\title{
A Retrospective Quasi-Experimental Study of a Community Crisis House for Patients with Severe and Persistent Mental Illness
}

Running Title: Alternative to Hospitalisation Evaluation

Authors: Dan Siskind ${ }^{*}$, Lecturer ${ }^{1}$, Senior Lecturer ${ }^{2}$, and Consultant Psychiatrist ${ }^{3}$ Meredith Harris, Senior Research Fellow ${ }^{1}$,

Steve Kisely, Professor ${ }^{1,2,3,4,5}$

James Brogan, Research Assistant ${ }^{1,3}$

Jane Pirkis, Professor ${ }^{6}$,

David Crompton, Associate Professor ${ }^{2}$, and Executive Director ${ }^{3}$

Harvey Whiteford, Kratzmann Professor ${ }^{1}$

1 School of Population Health, The University of Queensland, Qld, Australia

2 School of Medicine, The University of Queensland, QId, Australia

3 Metro South Division of Mental Health, Woolloongabba, Qld, Australia

$4 \quad$ Health LinQ, University of Queensland, St Lucia, QId, Australia

$5 \quad$ Griffith Institute of Health, Griffith University, QId, Australia

6 Centre for Health Policy, Programs and Economics, School of Population Health, University of Melbourne, Melbourne, Vic, Australia

*corresponding author

Queensland Centre for Mental Health Research

Level 3 Dawson House

The Park

Wacol QLD 4076

AUSTRALIA

dan siskind@gcmhr.uq.edu.au

mobile +61420680250

office +61732718660

fax

+61732718698

Submitted to:

Australian and New Zealand Journal of Psychiatry

Original Submission date: 13 September 2012

Resubmission date: 15 February 2013 


\begin{abstract}
Objective: There is increasing international evidence that crisis houses can reduce the time spent in acute psychiatric inpatient units for patients with severe and persistent mental illness, at a lower cost and in an environment preferable to patients. We evaluated the Alternatives to Hospitalisation (AtH) program, a crisis house operating in outer suburban Brisbane.

Method: 193 AtH patients were compared to 371 matched controls admitted to a peer hospital district acute psychiatric unit. Hospitalisations, demographics and illness acuity were compared one year before and after an acute index episode of residential care involving hospital and/or AtH. Hospital bed days during the index episode were compared between AtH participants and controls. The cost of bed days averted was compared to the cost of providing the AtH program.
\end{abstract}

Results: AtH participants spent 5.35 fewer days in hospital during the index episode than controls, after adjustment for illness acuity, living conditions, marital status and ED presentations. Per patient cost of averted psychiatric inpatient bed days, \$5948.22, was higher than the per patient cost of providing $\mathrm{AtH}, \$ 3071.44$. AtH participants had higher levels of illness acuity, ED presentations and acute psychiatric admissions than controls in the year after the index episode.

Conclusions: For acutely unwell, stably housed patients, able to be managed outside of a secure facility, a crisis house program can reduce acute psychiatric bed days, providing a cost saving for mental health services.

Keywords: Mental Health; Service Evaluation; Hospital; Crisis Housing 


\section{Introduction}

Acute psychiatric inpatient units may not provide a safe, therapeutic environment for patients (Muijen 1999), and are often not well regarded by consumers (Quirk, \& Lelliott 2001). Reductions in acute hospital beds per capita (Department of Health and Ageing 2007) and an increasing focus on psychosocial rehabilitation (Council of Australian Governments (COAG) 2006) and enhancing social inclusion have led current policy makers to see community crisis houses as a preferred mode of treatment for consumers with severe and persistent mental illness (SPMI) who experience acute exacerbations requiring acute residential level of care (Davies et al 1994; Johnson et al 2007; Thornicroft, \& Bebbington 1989; Andrews, \& Titov 2007).

Crisis houses are 24 hour staffed home-like facilities in the community, providing short term accommodation while patients in crisis can have their illness stabilised (Siskind et al 2012b). They aim to shorten psychiatric inpatient admissions and avert hospitalisations by providing an alternative to inpatient treatment. Outpatients with increased illness acuity can enter as a step-up from community care, with the aim of averting a psychiatric admission, while inpatients still requiring residential level of care can step-down from inpatient units to crisis houses, reducing hospital length of stay (Goodwin, \& Lyons 2001).

A 2009 systematic review of residential alternatives to acute psychiatric hospital admission in North America and Europe noted three models of community based crisis houses described in the literature (Lloyd-Evans et al 2009). The first was the "Soteria" model for people with early psychosis, which employed non-clinical staff working 36-48 hour shifts, had staff and residents sharing household tasks, and had reduced use of anti-psychotic medications (Mosher, \& Menn 1978; Fenton et al 1998). The second, a crisis house service in the UK, was co-located with a community mental health service, with clinical staff and psychiatrist supervision (Boardman et al 1999). The third, based in the USA, was a crisis service for Veterans operating out of houses in the community, staffed by clinicians and psychiatrists who are are linked to Veterans Affairs psychiatric hospitals (Hawthorne et al 2005).

Factors that contribute to whether a consumer in crisis will require hospitalization include danger to self, severity of psychosis, ability to self care, impulse control and depression severity (Way, \& Banks 2001). Crisis houses, unlike inpatient units, are 
not designed to provide care for severely agitated patients or for patients at risk of harming themselves or others (Way, \& Banks 2001; Lloyd-Evans et al 2009). The severity of these factors varies between consumers in crisis. Thus appropriate patient selection is important. Crisis houses may be more suitable for patients known to the mental health service, with less impaired ability to self care and greater impulse control, and who are at lower risk of harm to themselves and others.

The 2009 systematic review of crisis houses found that patients generally preferred community crisis houses over inpatient care, and that crisis houses were less costly than inpatient care (Lloyd-Evans et al 2009). A Queensland snapshot evaluation of all public psychiatric inpatients suggested that if sufficient crisis accommodation for people with severe and persistent mental illness was available, $18 \%$ of admitted patients could be discharged (Mental Health Branch, Queensland 2009). A New Zealand study has suggested that $12 \%$ of patients with SPMI presenting to emergency departments requiring admission could be diverted to crisis houses (Abas et al 2003).

Given the increasing role crisis houses play in mental health services in Australia, we undertook an evaluation of a crisis house service, Alternatives to Hospitalisation $(\mathrm{AtH})$, operating in an outer metropolitan hospital district of Brisbane.

In this study, we examined the effect of AtH for patients with SPMI during an acute index illness episode requiring residential level of care on psychiatric hospital bed days required and service costs during the acute episode, and clinical and service usage outcomes in the year following discharge. We compared patients who accessed AtH with a matched comparison group drawn from a peer mental health service without access to a crisis house service. We examined whether:

1. the availability of a crisis house program would reduce the number of hospital bed days required by patients during an acute illness exacerbation requiring residential level of care

2. the cost of providing AtH would be less than the cost of the averted hospital bed days

3. there was a difference between AtH participants and controls in the year after the acute index episode on clinical and service usage measures (psychiatric hospital readmissions, emergency department (ED) presentations, illness 
acuity, problems with living conditions, substance abuse, self harm and mortality).

4. Step-Up and Step-Down AtH participants differed in terms of duration in hospital during the index episode, or on clinical and service usage measures for the year after the index episode. 


\section{Methods}

Setting

AtH services were provided to a general hospital district with a catchment population of 300,000 residents in the outer southern suburbs of Brisbane. The hospital has an integrated mental health service (Thornicroft, \& Tansella 2004). Services include an acute inpatient psychiatric unit, community based multi-disciplinary outreach clinical case management teams with a staff to patient ratio of approximately 1:25 (Harvey, \& Fielding 2003), an Assertive Community Treatment model team (Harvey et al 2012), a psychiatric emergency department and a home based mobile acute crisis team (Johnson et al 2005). Further details on mental health services in Queensland is available elsewhere (Harris et al 2012; Siskind et al 2012a).

\section{Program Description}

AtH operated a four bedroom crisis house service with five beds on a residential suburban street in outer Metropolitan Brisbane. The house was not distinguishable from other houses on the street. Three patients had individual rooms, while two patients shared a room.

Entry criteria for AtH included: having severe and persistent mental illness; an illness acuity score below 40 on the global assessment of functioning (GAF) scale, (e.g. serious impairment in judgement, thinking, mood or reality testing) (Jones et al 1995); being a case managed patient of the hospital district; having a place to live after exit from $\mathrm{AtH}$; and a willingness to enter the program (irrespective of whether patient is under an involuntary treatment order). Exclusion criteria included: active suicide risk; recent history of violence; extreme disorganisation; and high risk of substance withdrawal.

Patients could stay for up to two weeks and could have their stay briefly extended on a case by case basis.

There was 24 hour on-site staffing by enrolled nurses, with additional on-site staffing from a clinical nurse and an occupational therapist from $8 \mathrm{am}$ to $4 \mathrm{pm}$, Monday to Friday. The service model was based on the principles of psychosocial rehabilitation (Anthony 1993). Consumers were expected to contribute to the running of the house, including assisting with cooking of shared meals and cleaning. Patients were 
involved in creating recovery plans and crisis and relapse prevention strategies. With patient consent, families and carers were actively involved in care planning for patients, and were provided psycho-education and referral to community supports. Patients were assisted to link with community services such as clubhouses, employment services and mental health support worker services.

AtH was integrated into the hospital district community mental health service. AtH team members were involved in service wide education and training activities, sat on service wide committees, and were in regular contact with clinical inpatient and outpatient teams. AtH participants continued to receive clinical case management as usual, which included assessment, monitoring and treatment planning from their geographical catchment continuing care team. Psychopharmacology was coordinated by the psychiatrists attached to the AtH participant's usual case management team.

\section{Study design and participants}

This was a retrospective quasi-experimental study comparing an intervention group to a control group.. The intervention group were all AtH program participants from the hospital district who consecutively entered the program for the first time after 1 April 2006 and exited before 31 March 2009. Controls were drawn from patients admitted to a demographically similar peer outer suburban hospital district in northern Brisbane that did not provide a crisis house service, during the same period.

\section{Data sources}

The intervention group and controls were identified from a set of linked, de-identified, administrative datasets: Consumer Integrated Mental Health Application (CIMHA); Client Event Services Application (CESA); Outcome Information System (OIS); Emergency Department Information System (EDIS); Queensland Hospital Admitted Patient Data Collection (QHAPDC); and the Queensland Registrar General Mortality dataset. These datasets capture information about all patients seen by public mental health services in Queensland.

Data were extracted on the intervention group and controls for the year before the index date (defined as the date of admission for the hospitalization immediately preceding a participant's first entry into AtH for Step-Down patients, the date of first 
entry into AtH for Step-Up patients, and the date of the index admission to hospital in the study period (1 April 2006 - 31 March 2009) for controls), and for a year following the exit date (defined as the date a intervention group member exited their first stay in $\mathrm{AtH}$ accommodation, or the date a control was discharged from hospital).

\section{Control selection}

The variables used to match controls to the intervention group were gender, period of entry grouped into 6 half year time periods: 1 April to 30 September and 1 October to 31 March; and diagnosis, in three categories: psychosis (ICD 10 codes F20-29); affective disorders (F30-39); and other disorders (including anxiety (F40-43, personality disorders (F60), pervasive developmental disorders (F84) and substance use disorders (F10-19)). A three-stage process was used to select controls. In the first stage, all patients with an admission to the peer hospitals from 1 April 2006 to 30 March 2009 were included in a list of potential controls. In the second stage, using SPSS version 20 "FUZZY" plugin, AtH participants were randomly matched to up to 4 controls from the stage one list on index date, gender and diagnosis category to create a control group pool. Of this control group pool only $24.4 \%$ had admissions in the year prior to the index date, compared to $47.2 \%$ among the intervention group $\left(\chi^{2}=37.68\right.$, d.f. $\left.=1, p<0.001\right)$. To reduce the risk of this disparity confounding the analysis, in the third stage, a sub-group of controls was randomly selected from the control group pool so as to contain the same proportion of persons with admissions in the year prior to the index date as did the AtH participant group. All further analyses were conducted using this third stage control sub-group, hereafter referred to as the controls.

\section{Measures Used}

The outcome variables investigated were: acute psychiatric hospitalisations; illness acuity, problems with living conditions, suicidality and substance abuse as measured by the Health of the Nation Outcome Scale (HoNOS); emergency department (ED) presentations; mortality; and re-entry to AtH for AtH participants.

The index episode was defined as the period of acute illness requiring residential level of care, either hospital or $\mathrm{AtH}$, commencing at the index date and ending with exit from $\mathrm{AtH}$ for $\mathrm{AtH}$ participants, and discharge from hospital for controls. We calculated the inpatient bed days during the index episode for $\mathrm{AtH}$ participants and 
controls. For Step-Down AtH patients, this comprised hospitalisation after the index date that ended when the patients entered AtH. Step-up AtH patients entered the program directly from the community, averting an index hospitalisation, and as such had no hospital bed days during the index episode. As controls spent the entire index episode in hospital, the duration of hospitalisation was the same as the duration of the index episode.

Hospital bed-days in the year before the index date and the year after exit were calculated as the cumulative number of nights spent in hospital for all psychiatric hospitalisations in the year before the index and the year after exit.

The Health of the Nation Outcome Scale (HoNOS) is a 12 question instrument that measures symptoms and social function (Wing et al 1998). Multiple HoNOS ratings could be available for a given patient in each time period. We used the highest HoNOS rating in the year before the index date and in the year after exit. The total HoNOS score was used as an outcome measure of overall illness acuity. Higher scores indicate higher acuity. Three of the component questions were used to measure: suicidal ideation, question two (Q2); substance use, question three (Q3); and problems with living conditions, question 11 (Q11). The individual questions are rated $0-4$, with scores of 2 and above considered to be clinically significant (Burgess et al 2009). Dichotomizing the scores for the individual questions to below two versus two or above allowed the calculation of rates of clinically significant problems. The measure of ED presentations was the total number of presentations to ED for any condition in the year before the entry date and the year after exit. Days open to case management after the exit date was calculated as the number of days the patient's file was open between the exit date and the closure of the clinical file. All patient's with open files were assumed to be open to case manangement. The date of closure of the clinical file was ascertained from the electronic records. If the file was still open a year after the end of the index episode, the days open to case management was set at 365 days. Data on mortality was obtained through data linkage to the Queensland Registrar General Mortality dataset.

For AtH participants, data on re-entry into $\mathrm{AtH}$ for the year after exit were collected. Covariates used included age, marital status (married/defacto vs single), diagnoses (psychosis, affective disorders and other which included anxiety disorders, 
personality disorders and substance abuse), indigenous status, and date of index episode.

\section{Statistical analysis}

Demographics and Service Use

For the year prior to the index date, data for AtH participants and controls were compared on demographics, psychiatric hospitalisations, ED presentations, illness acuity, problems with living conditions, substance abuse and suicidal ideation using t-tests or $\chi^{2}$, as appropriate.

Index Episode Bed Days

An unadjusted negative binomial regression model was used to compare the number of hospital bed days for the index episode between the intervention group and controls. An adjusted negative binomial regression model, accounting for covariates for which there were differences between AtH and participants in the year prior to the index episode, was used to calculated the estimated marginal mean hospital bed days for the index episode. Estimated marginal means of the hospital bed days for the index episode were derived by holding covariates at their mean value.

Estimated marginal means is a statistical method of comparing the means of two groups that have been adjusted for covariates. A negative binomial regression model is a statistical method for examining predictive relationships with a count dependent model. It can be used with non-normal heteroscedastic distributions and over-dispersed count variables (Elhai et al 2008). Negative binomial is particularly useful for count variables with a high number of zero values such as bed days, where many participants are never admitted to hospital and have a bed day count of zero.

\section{Cost Analysis}

A cost analysis was undertaken using a cost-minimisation approach. Using hospital inpatient pricing data, the cost of a psychiatric inpatient bed-day in 2009 was estimated to be $\mathrm{AU} \$ 1111.82$. The per-diem cost of AtH was $\$ 305.02$, derived by dividing the total annual program costs for 2008-09 by the number of available 
places per day. The per patient cost of the bed days averted for AtH participants during the index episode was compared to the per participant cost of $\mathrm{AtH}$.

\section{$\underline{\text { AtH Participants }}$}

The mean duration of days in AtH in the index episode was compared between StepUp and Step-Down patients using a t-test.

Outcomes in Year After Exit

Outcomes in the year after the exit date, including rehospitalisations, illness acuity, problems with living conditions, substance abuse, suicidal ideation, mortality and duration open to case management were compared using t-tests, or $\chi^{2}$ as appropriate.

All analyses were conducted using SPSS for Mac, version 20 (Chicago, IL: SPSS Inc., 2011).

\section{Ethics}

Ethics approval was granted from the Metro South Human Research Ethics Committee (HREC), University of Queensland School of Population Health Research Ethics Committee and Queensland Health Research and Governance Unit. 


\section{Results}

Demographic, service utilisation and clinical characteristics of participants

Summary characteristics for AtH participants and matched controls are presented in Table 1. 193 patients had first admissions to AtH during the study period and were included in the analysis. Using the three stage matching protocol, the first stage potential control group was reduced from 2,877 to 689 in stage two, and to 371 in stage three. All further analyses were conducted using the 371 patient stage three control group, hereafter referred to as the controls.

There was no statistically significant difference between AtH participants and controls on age, gender, diagnosis, indigenous status, index date, rate of psychiatric admission in the year before index date, mean bed days in the year before index date for admitted patients or rates of clinically significant substance use and non accidental self harm. AtH participants were more likely to be married or in a de-facto relationship, had a lower total HoNOS score in the year before the index date, a lower rate of clinically significant problems with living conditions, and a higher rate of ED presentations than controls. (Table 1)

Table 1 about here

\section{Index Episode Bed Days}

Using a negative binomial regression model, the duration of AtH participant hospital bed days in the index episode were $69.4 \%$ of those of control admissions, with an estimated marginal mean admission duration of 14.67 days (S.E. 1.30) for AtH participants and 21.15 days (S.E. 1.34) for controls (Table 2). When adjusted for marital status, highest HoNOS, rate of ED presentations and problems with living conditions in the year before the index date using a negative binomial regression model, AtH participant hospital bed days in the index episode were $76.9 \%$ of the duration of those of controls, with an estimated marginal mean admission duration of 14.42 days (S.E. 2.00) for AtH participants and 19.77 days (S.E. 2.20) for controls (mean total HoNOS score fixed at 17.13). The mean inpatient bed days in the index episode were therefore 6.48 days shorter for AtH participants in the unadjusted model, and 5.35 days shorter for AtH participants after adjustment for marital status, highest HoNOS, rate of ED presentations and problems with living conditions. 
The mean duration in AtH for AtH participants during the index episode was 9.45 days (S.E. 0.44). The mean duration of the total index episode was 24.12 days for AtH participants and 22.87 days for controls $(t=-0.34$, d.f. $=562, p<0.001)$.

AtH participants entering the program as a "Step-Up" from community care made up $34.7 \%$ of the total group. Mean duration in AtH was not statistically significantly different for the Step-Up (9.91 days, S.E. 0.87) or Step-Down (9.20 days, S.E. 0.50) groups $(t=-0.765$, d.f. $=191, p=0.445)$. There was no statistically significant difference between AtH Step-Up or Step-Down participants on rates of re-hospitalisation, reentry into $\mathrm{AtH}$, or ED presentations in the year after the end of the index episode.

\section{$\underline{\text { Cost Analysis }}$}

AtH participants spent 5.35 fewer days in hospital during the index episode than controls after adjustment for illness acuity, ED presentations, marital status and problems with living conditions. This lead to a reductions in costs per patient to the mental health service of $A \cup \$ 5948.22$. This was higher than the cost to the mental health service for the AtH program of AU\$3071.44 per participant.

\section{AtH participants and Controls in the year after end of index episode}

In the year after exit, AtH participants were significantly more likely to be readmitted to hospital than controls, although mean bed days for admitted patients were not significantly different (Table 3). AtH participants had a higher total HoNOS score in the year after the end of the index episode, a higher rate of clinically significant problems with living conditions, a higher rate of ED presentations, and a longer duration open to the mental health service as a case managed patient, than controls. There was no significant difference in rates of clinically significant problems with substance use or non-accidental self harm in the year after the end of the index episode.

\section{Table 3 about here}

Twenty-three percent of AtH participants had a re-entry to AtH within a year of exit. When re-entry to AtH within a year of exit was combined with hospital readmission within year of exit, rates of admission for AtH participants increased from $46.1 \%$ for 
hospital re-admission to $53.4 \%$ for re-admission to either AtH or hospital (McNemar test $p=0.002$ ). 


\section{Discussion}

AtH participants spent fewer days in hospital during the index episode when compared to controls, after adjustment for illness acuity, marital status, rate of ED presentations and living conditions. This led to a cost savings to the mental health service.

This study is one of the few studies of crisis houses internationally to include a cost analysis.

This study used a retrospective quasi-experimental design, and included all $\mathrm{AtH}$ participants using the program for the first time. There were no research exclusion criteria, allowing the analysis to reflect real world practice.

The data used in this study were sourced from de-identified administrative dataextracts of all public mental health patients in Queensland. There are inherent limitations with administrative data, including missing data and risk of recording bias. The datasets used primarily for billing, including inpatient data and ED presentations, provided close to a complete capture of data. HoNOS data were available for over $82 \%$ of all patients across all time periods.

The availability of community services is a predictor of length of stay in acute psychiatric hospitals (Zhang et al 2011). The hospitals in this study both served similar population demographics in outer southern and northern suburban Brisbane, and provided a similar suite of case management and community mental health services. Although it is possible there were differences between the mental health services, the three stage randomised control matching process was designed to reduce differences between the AtH participants and controls and reduce potential confounders.

An advantage of a retrospective quasi-experimental study is the exploration of differences between the AtH participants and the broader population accessing acute psychiatric inpatient services. This provides information to services planners on the patient population using $\mathrm{AtH}$, and can inform further research on appropriate target populations for crisis house services.

There were demographic and service usage differences between the AtH participants and controls. AtH participants had lower levels of illness acuity than controls in the year before the index date, in keeping with the program's entry 
criteria. AtH participants had lower rates of clinically significant problems with living conditions compared to controls, as entry into $\mathrm{AtH}$ was predicated on having an established safe place to live after discharge. AtH participants mere more likely to be married or in a defacto relationship than controls, which may be a proxy measure for social stability.

These differences between AtH participants and controls had the potential to confound unadjusted comparisons between the groups. To address this we adjusted the negative binomial regression of inpatient bed days in the index episode for illness acuity, marital status, rates of ED presentations and problems with living conditions in the year before the index date.

We did not have data on whether patients were under involuntary treatment orders (ITO) during the course of the index episode. In Queensland, there are two categories of ITO, inpatient and community. Inpatient category ITO patients require treatment in a gazetted mental health inpatient facility, though they can be given leave to temporarily reside in a facility such as AtH. Community ITO patients do not have restrictions on where they can reside. A requirement of entry into $\mathrm{AtH}$ was a willingness to engage with the AtH program, but being under an ITO was not a barrier to entry. It is possible that the control population had a higher rate of inpatient ITOs, and that this may have lead to undetected differences between AtH participants and controls. Although there is a risk that this potential difference could confound the analysis, a Cochrane systematic review found that ITOs did not alter service use (Kisely et al 2011). Illness acuity may provide a proxy measure for involuntary treatment (Xiao et al 2004), and was included in the adjusted negative binomial regression.

\section{AtH participants differed from controls in the year after the index episode on several} outcome measures, including rates of acute psychiatric admission, illness acuity, ED presentations and days open to case management. In the year after exit, $\mathrm{AtH}$ participants were more likely than controls to have an acute psychiatric admission in the year after exit. AtH participants experienced little change in their HoNOS score in the year before and after the program, and had higher rates of ED presentations than controls in the year after exit. They had more days open to case management in the year after the end of the index episode. The increased acute service usage for 
$\underline{\text { AtH participants in the year after exit would lead to higher costs to the mental health }}$ service.

Being open to case management was-significantly correlated with having a psychiatric hospitalisation in the year after the end of the index episode $\left(x^{2}=36.82\right.$, $p<0.001)$. The increased time open to case management for the AtH participants would increase the cost to the mental health service.

It is possible that the increased duration open to case management and rate of rehospitalisation is due to the AtH program creating greater dependency on the mental health service. However, it is more likely that the increased service usage is related to the $\mathrm{AtH}$ arm of the study selecting for patients already open to case management. Case managed patients have been shown to have higher rates of rehospitalisation and service contact (Ziguras, \& Stuart 2000). Among the patients evaluated in our study, $\underline{\text { Bb}}$ eing open to case management was significantly correlated with having a psychiatric hospitalisation in the year after the end of the index episode $\left(x^{2}=36.82, p<0.001\right)$.

Given the target population of ATH was patients currently open to case management, it would be reasonable to expect a trajectory of illness that would continue after exit from ATH, including greater illness acuity, emergency department presentations, readmissions and continued case management. We were unable to test this hypothesis as we were unable to adjust the analysis for whether patients were open to case management in the year prior to the index date. Further evaluations of crisis house programs are required to assist in clarifying whether higher illness acuity and acute service usage in the year after exit is related to program effect or a confounder associated with the target population.

\section{Comparison to other crisis house evaluations}

Three crisis house evaluations from the US and UK found that number of readmissions (Hawthorne et al 2005) and illness acuity (Hawthorne et al 2005; Fenton et al 2002; Fenton et al 1998; Mosher et al 1995) were not significantly different at follow up ranging from 2 to 6 months for patients randomly assigned to 
crisis house or inpatient psychiatric facility. As our study used a retrospective quasiexperimental design, we were not able to prospectively control for chronicity and acuity, which may explain the difference in results.

In a study from the US, Fenton et. al. (2002), the duration of the residential alternative program was longer than standard inpatient care (12 vs 19 days $p<0.001$ ) (Fenton et al 2002; Fenton et al 1998). This was in keeping with the duration of the index episode in our study, which was longer for AtH participants.

\section{Crisis House program evolution and research}

Our evaluation identified differences between the population using $\mathrm{AtH}$ and the broader patient group accessing acute psychiatric inpatient services. Further research is needed to identify which patient groups gain most benefit from crisis house services. This research has the potential to aid service planners to identify the most appropriate patient populations for referral to crisis houses.

When considering the scaling up of mental health interventions, it is important to consider whether the results of an evaluation are generalisable to the broader target population. This evaluation was of a single site, in an outer suburban Australian public integrated mental health service. As this was a retrospective evaluation using administrative data, it is unlikely that the results are due to a "Hawthorne" effect (Parsons 1974). Adjustments to the service model would be required to contextualise the program to other mental health services with different population demographics and needs.

\section{Conclusions}

Crisis houses such as AtH are not appropriate for all patients. This program was targeted at patients with chronic mental illness with lower levels of illness acuity, who did not have active suicide or violence risk, and could be managed outside of a secure facility. In Australia, the proposed introduction of activity based funding for mental health will have an impact on the funding for inpatient mental health services. This may lead to financial disincentives for extended duration admissions. Programs such as AtH that can shorten or avert psychiatric hospitalisations for patients with frequent hospital presentations and chronic, lower illness acuity have the potential to deliver cost savings to mental health services, while providing a home-like environment preferred by patients. 


\section{Acknowledgements}

The authors acknowledge the Queensland Directorate of Mental Health for access to de-identified data extracts and assistance in data linkage, Dr Victor Siskind for statistical advice, Dr Cathy Mihalopoulos for advice on economic analysis and the staff of the Alternatives to Hospitalisations Team. 


\section{References}

Abas, M., Vanderpyl, J., Le Prou, T., Kydd, R., Emery, B. \& Foliaki, S.A., 2003, Psychiatric hospitalization: reasons for admission and alternatives to admission in South Auckland, New Zealand, The Australian and New Zealand journal of psychiatry, 37(5), pp. 620-5.

Andrews, G. \& Titov, N., 2007, Changing the face of mental health care through needs-based planning, Australian health review : a publication of the Australian Hospital Association, 31 Suppl 1, pp. S122-8.

Anthony, W.A., 1993, Recovery from Mental Illness: The Guiding Vision of the Mental Health Service System in the 1990s, Psychosocial Rehabilitation Journal, 16(4), pp. 11-23.

Boardman, A.P., Hodgson, R.E., Lewis, M. \& Allen, K., 1999, North Staffordshire Community Beds Study: Iongitudinal evaluation of psychiatric in-patient units attached to community mental health centres. I: Methods, outcome and patient satisfaction, The British journal of psychiatry : the journal of mental science, 175, pp. 70-8.

Burgess, P., Trauer, T., Coombs, T., McKay, R. \& Pirkis, J., 2009, What does 'clinical significance' mean in the context of the Health of the Nation Outcome Scales? Australasian psychiatry : bulletin of Royal Australian and New Zealand College of Psychiatrists, 17(2), pp. 141-8.

Council of Australian Governments (COAG), 2006, National Action Plan on Mental Health 2006-2011, Canberra.

Davies, S., Presilla, B., Strathdee, G. \& Thornicroft, G., 1994, Community beds: the future for mental health care? Social psychiatry and psychiatric epidemiology, 29(6), pp. 241-3.

Department of Health and Ageing, 2007, National Mental Health Report 2007: Summary of Twelve Years of Reform in Australia's Mental Health Services under the National Mental Health Strategy 1993-2005, Commonwealth of Australia,.

Elhai, J.D., Calhoun, P.S. \& Ford, J.D., 2008, Statistical procedures for analyzing mental health services data, Psychiatry research, 160(2), pp. 129-36. 
Fenton, W.S., Hoch, J.S., Herrell, J.M., Mosher, L. \& Dixon, L., 2002, Cost and costeffectiveness of hospital vs residential crisis care for patients who have serious mental illness, Archives of General Psychiatry, 59(4), pp. 357-64.

Fenton, W.S., Mosher, L.R., Herrell, J.M. \& Blyler, C.R., 1998, Randomized trial of general hospital and residential alternative care for patients with severe and persistent mental illness, American Journal of Psychiatry, 155(4), pp. 516-22.

Goodwin, R. \& Lyons, J.S., 2001, An emergency housing program as an alternative to inpatient treatment for persons with severe mental illness, Psychiatric services (Washington, D.C.), 52(1), pp. 92-5.

Harris, M.G., Buckingham, W.J., Pirkis, J., Groves, A. \& Whiteford, H., 2012, Planning estimates for the provision of core mental health services in Queensland 2007 to 2017, The Australian and New Zealand journal of psychiatry, 46(10), pp. 982-94.

Harvey, C., Killaspy, H., Martino, S. \& Johnson, S., 2012, Implementation of assertive community treatment in Australia: model fidelity, patient characteristics and staff experiences, Community mental health journal, 48(5), pp. 652-61.

Harvey, C.A. \& Fielding, J.M., 2003, The configuration of mental health services to facilitate care for people with schizophrenia, The Medical journal of Australia, 178 Suppl, pp. S49-52.

Hawthorne, W.B., Green, E.E., Gilmer, T., Garcia, P., Hough, R.L., Lee, M., Hammond, L. \& Lohr, J.B., 2005, A randomized trial of short-term acute residential treatment for veterans, Psychiatric services (Washington, D.C.), 56(11), pp. 1379-86. Johnson, S., Gilburt, H., Lloyd-Evans, B. \& Slade, M., 2007, Acute in-patient psychiatry: residential alternatives to hospital admission, Psychiatric Bulletin, 31, pp. 262-4.

Johnson, S., Nolan, F., Pilling, S., Sandor, A., Hoult, J., McKenzie, N., White, I.R., Thompson, M. \& Bebbington, P., 2005, Randomised controlled trial of acute mental health care by a crisis resolution team: the north Islington crisis study, BMJ (Clinical research ed.), 331(7517), p. 599. 
Jones, S.H., Thornicroft, G., Coffey, M. \& Dunn, G., 1995, A brief mental health outcome scale-reliability and validity of the Global Assessment of Functioning (GAF), The British Journal of Psychiatry, 166(5), pp. 654-9.

Kisely, S.R., Campbell, L.A. \& Preston, N.J., 2011, Compulsory community and involuntary outpatient treatment for people with severe mental disorders, Cochrane database of systematic reviews (Online), 2.

Lloyd-Evans, B., Slade, M., Jagielska, D. \& Johnson, S., 2009, Residential alternatives to acute psychiatric hospital admission: systematic review, The British journal of psychiatry : the journal of mental science, 195(2), pp. 109-17.

Mental Health Branch, Queensland, 2009, Queensland Mental Health Inpatient Snapshot Survey 2008 Report, Queensland Government Mental Health Branch. Mosher, L.R. \& Menn, A.Z., 1978, Community residential treatment for schizophrenia: Two-year follow-up, Hospital and Community Psychiatry, 29(11), pp. 715-23.

Mosher, L.R., Vallone, R. \& Menn, A., 1995, The treatment of acute psychosis without neuroleptics: six-week psychopathology outcome data from The Soteria Project, The International journal of social psychiatry, 41(3), pp. 157-73.

Muijen, M., 1999, Acute hospital care: ineffective, inefficient and poorly organised, Psychiatric Bulletin (),, 257-259, 23(5), pp. 257-9.

Parsons, H.M., 1974, What happened at Hawthorne? Science, 183(4128), pp. 92232.

Quirk, A. \& Lelliott, P., 2001, What do we know about life on acute psychiatric wards in the UK? A review of the research evidence, Social science \& medicine (1982), 53(12), pp. 1565-74.

Siskind, D., Harris, M., Buckingham, B., Pirkis, J. \& Whiteford, H., 2012a, Planning estimates for the mental health community support sector, The Australian and New Zealand journal of psychiatry, 46(6), pp. 569-80.

Siskind, D., Harris, M., Pirkis, J. \& Whiteford, H., 2012b, A domains-based taxonomy of supported accommodation for people with severe and persistent mental illness, Social psychiatry and psychiatric epidemiology, accepted 5/9/12(in press). 
Thornicroft, G. \& Bebbington, P., 1989, Deinstitutionalisation--from hospital closure to service development, The British journal of psychiatry : the journal of mental science, 155, pp. 739-53.

Thornicroft, G. \& Tansella, M., 2004, Components of a modern mental health service: a pragmatic balance of community and hospital care: overview of systematic evidence, The British journal of psychiatry : the journal of mental science, 185, pp. 283-90.

Way, B.B. \& Banks, S., 2001, Clinical factors related to admission and release decisions in psychiatric emergency services, Psychiatric services (Washington, D.C.), 52(2), pp. 214-8.

Wing, J.K., Beevor, A.S., Curtis, R.H., Park, S.B., Hadden, S. \& Burns, A., 1998, Health of the Nation Outcome Scales (HoNOS). Research and development, The British journal of psychiatry : the journal of mental science, 172, pp. 11-8.

Xiao, J., Preston, N.J. \& Kisely, S., 2004, What determines compulsory community treatment? A logistic regression analysis using linked mental health and offender databases, The Australian and New Zealand journal of psychiatry, 38(8), pp. 613-8. Zhang, J., Harvey, C. \& Andrew, C., 2011, Factors associated with length of stay and the risk of readmission in an acute psychiatric inpatient facility: a retrospective study, The Australian and New Zealand journal of psychiatry, 45(7), pp. 578-85.

Ziguras, S.J. \& Stuart, G.W., 2000, A meta-analysis of the effectiveness of mental health case management over 20 years, Psychiatric Services, 51(11), pp. 1410-21. 


\section{Tables}

Table 1 Demographic, service utilization and clinical characteristics of AtH participants and controls

[Percentage and number unless otherwise stated]

\begin{tabular}{|c|c|c|c|c|}
\hline Demographic & $\begin{array}{r}\text { AtH } \\
\text { participants }\end{array}$ & Controls & t-test $/ \chi^{2}$ & $\mathbf{p}$ \\
\hline Number & 193 & 371 & & \\
\hline Age, years (mean (SE)) & $37.60(0.84)$ & $36.91(0.60)$ & $-0.68^{\dagger}$ & 0.498 \\
\hline Male & $41.5 \%(80)$ & $41.0 \%(152)$ & $0.01^{\ddagger}$ & 0.912 \\
\hline \multicolumn{5}{|l|}{ Diagnosis } \\
\hline Psychosis & $43.0 \%(83)$ & $46.6 \%(173)$ & $0.89^{\S}$ & 0.640 \\
\hline Affective disorder & $32.1 \%(62)$ & $31.5 \%(117)$ & & \\
\hline Other ${ }^{\|}$ & $24.9 \%(48)$ & $21.8 \%(81)$ & & \\
\hline Married or defacto & $25.4 \%(49 / 193)$ & $8.8 \%(30 / 340)$ & $26.76^{\ddagger}$ & $<0.001^{* * *}$ \\
\hline Indigenous & $5.7 \%(11)$ & $7.0 \%(26)$ & $0.36^{\ddagger}$ & 0.551 \\
\hline Index date in first half of study period & $48.2 \%(93)$ & $50.1 \%(186)$ & $0.19^{\ddagger}$ & 0.661 \\
\hline Any admission in the year before the index date & $47.2 \%(91)$ & $45.0 \%(167)$ & $0.23^{\ddagger}$ & 0.629 \\
\hline Bed Days of admitted patients in the year before the index date (mean (SE)) & $26.14(3.72)$ & $27.85(3.44)$ & $0.32^{\dagger \dagger}$ & 0.753 \\
\hline Highest HoNOS in the year before the index date (mean (SE)) & $14.64(0.57)$ & $18.35(0.38)$ & $5.45^{\ddagger \ddagger}$ & $<0.001^{* * *}$ \\
\hline Clinically significant problems with living conditions in the year before the index date $\$ \S$ & $31.3 \%(47 / 161)$ & $48.6 \%(157 / 323)$ & $12.46^{\ddagger}$ & $<0.001^{\ldots *}$ \\
\hline Clinically significant substance use in the year before the index date $\mathrm{e}^{\S \S}$ & $50.7 \%(76 / 160)$ & $55.2 \%(175 / 317)$ & $0.84^{\ddagger}$ & 0.358 \\
\hline Clinically significant non-accidental self harm in the year before the index date ${ }^{\S \S}$ & $39.2 \%(60 / 162)$ & $41.1 \%(1139 / 338)$ & $0.16^{\ddagger}$ & 0.690 \\
\hline Any ED presentation in the year before the index date & $88.6 \%(171)$ & $75.2 \%(279)$ & $14.13^{\ddagger}$ & $<0.001^{* * x}$ \\
\hline
\end{tabular}


$\ddagger-\chi^{2}, \mathrm{df}=1$

$\S-\chi^{2}, \mathrm{df}=2$

|| - Other Diagnoses includes Anxiety disorders, personality disorders and substance abuse

†† - t-test, $\mathrm{df}=257$

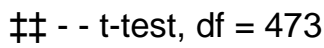

$\S \S$ - Number of patients with available data listed in the denominator in brackets

${ }^{* * *} p<0.001$, d.f. $=$ degrees of freedom, $E D=$ Emergency Department 
Table 2 Comparison of inpatient bed days during index episode for AtH Participants and Controls

\begin{tabular}{|c|c|c|c|c|c|c|}
\hline $\begin{array}{l}\text { Negative Binomial } \\
\text { Regression Model }\end{array}$ & $\begin{array}{l}\text { AtH Participants } \\
\text { E.M. mean (S.E.) }{ }^{\dagger}\end{array}$ & $\begin{array}{l}\text { Controls } \\
\text { E.M. mean (S.E.) }{ }^{\dagger}\end{array}$ & $\begin{array}{l}\text { Wald } \\
\chi^{2}\end{array}$ & d.f. $^{\ddagger}$ & Significance & $\begin{array}{l}\text { AtH participants } \\
\text { as } \% \text { of controls } \S\end{array}$ \\
\hline Unadjusted Model & $14.67(1.30)$ & $21.15(1.34)$ & 11.31 & 1 & $<0.001$ & $69.4 \%$ \\
\hline Adjusted Model" & $14.42(2.00)$ & $19.77(2.20)$ & 4.92 & 1 & 0.027 & $76.9 \%$ \\
\hline
\end{tabular}

† - Estimated marginal mean days of index acute psychiatric hospitalisation (Standard Error of Mean)

$\ddagger$ - degrees of freedom

$\S$ - Estimated marginal mean bed days of AtH participants as a percentage of the estimated marginal mean bed days of controls

II - Model adjusted for marital status, illness acuity, rate of ED presentations and problems with living conditions. 
Table 3 Outcomes in the year after the end of the index episode

[Percentage and number unless otherwise stated]

\section{Demographic}

Any hospital readmission

Bed-days for readmitted patients (mean (S.E.))

Highest HoNOS (mean (S.E.))

Clinically significant problems with living conditions

Clinically significant substance use

Clinically significant non-accidental self harm

Any ED presentation

Days open to case management after exit (mean (S.E.))

Death

$\dagger-\chi^{2}, \mathrm{df}=1$

$\ddagger$ - t-test, $\mathrm{df}=183$

$\S$ - t-test, $d f=481$

II - t-test, $\mathrm{df}=562$

${ }^{* * *} \mathrm{p}<0.001$

d.f. $=$ degrees of freedom, ED $=$ Emergency Department

\begin{tabular}{rrll} 
AtH participants & Controls & t-test $/ \chi^{2}$ & $\mathbf{p}$ \\
$46.1 \%(89 / 193)$ & $25.9 \%(96 / 371)$ & $23.59^{\dagger}$ & $<0.001^{* \star *}$ \\
$31.43(4.87)$ & $39.24(5.93)$ & $1.01^{\ddagger}$ & 0.314 \\
$14.50(0.63)$ & $12.77(0.46)$ & $-2.18^{\S}$ & 0.030 \\
$48.4 \%(77 / 159)$ & $14.5 \%(46 / 317)$ & $63.56^{\dagger}$ & $<0.001^{* \star *}$ \\
$47.2 \%(75 / 159)$ & $39.1 \%(126 / 322)$ & $2.82^{\dagger}$ & 0.093 \\
$28.9 \%(46 / 159)$ & $24.7 \%(82 / 332)$ & $1.00^{\dagger}$ & 0.318 \\
$60.1 \%(116 / 193)$ & $39.6 \%(147 / 371)$ & $21.08^{\dagger}$ & $<0.001^{* \star *}$ \\
$248.99(10.82)$ & $91.68(8.05)$ & $11.55^{\|}$ & $<0.001^{* * \star}$ \\
$3.1 \%(6 / 193)$ & $1.3 \%(5 / 371)$ & $2.06^{\dagger}$ & 0.151 \\
\hline
\end{tabular}

\section{AtH participants}

$47.2 \%(75 / 159)$

$28.9 \%(46 / 159)$

248.99 (10.82)

$1.3 \%(5 / 371)$ 
